\title{
Cryoprotective activities of FK20, a human genome-derived intrinsically disordered peptide against cryosensitive enzymes without a stereospecific molecular interaction
}

\author{
Naoki Matsuo ${ }^{\text {Equal first author, 1 }}$, Natsuko Goda ${ }^{\text {Equal first author, 1 }}$, Takeshi Tenno ${ }^{1,2}$, Hidekazu Hiroaki ${ }^{\text {Corresp. 1, } 2}$ \\ ${ }^{1}$ Graduate School of Pharmaceutical Sciences, Laboratory of Structural and Molecular Pharmacology, Nagoya University, Nagoya, AICHI, JAPAN \\ 2 BeCellBar, LLC., Nagoya, Aichi, Japan \\ Corresponding Author: Hidekazu Hiroaki \\ Email address: hiroaki.hidekazu@f.mbox.nagoya-u.ac.jp
}

Background. Intrinsically disordered proteins (IDPs) have been shown to exhibit cryoprotective activity toward other cellular enzymes without any obvious conserved sequence motifs. This study investigated relationships between the physical properties of several human genome-derived IDPs and their cryoprotective activities.

Methods. Cryoprotective activity of three human-genome derived IDPs and their truncated peptides toward lactate dehydrogenase (LDH) and glutathione S-transferase (GST) was examined. After the shortest cryoprotective peptide was defined (named FK20), cryoprotective activity of all-D-enantiomeric isoform of FK20 (FK20-D) as well as a racemic mixture of FK20 and FK20-D was examined. In order to examine the lack of increase of thermal stability of the target enzyme, the CD spectra of GST and LDH in the presence of a racemic mixture of FK20 and FK20-D at varying temperatures were measured and used to estimate $T_{m}$.

Results. Cryoprotective activity of IDPs longer than 20 amino acids was nearly independent of the amino acid length. The shortest IDP-derived 20 amino acid length peptide with sufficient cryoprotective activity was developed from a series of TNFRSF11B fragments (named FK20) was identified. FK20, FK20-D, and an equimolar mixture of FK20 and FK20-D also showed similar cryoprotective activity toward LDH and GST. $T_{m}$ of GST in the presence and absence of an equimolar mixture of FK20 and FK20-D are similar, suggesting that IDPs' cryoprotection mechanism stems partly from a molecular shielding effect rather than a direct interaction with the target enzymes. 
1 Cryoprotective activities of FK20, a human genome-

2 derived intrinsically disordered peptide against

3 cryosensitive enzymes without a stereospecific

4 molecular interaction

5

$9 \quad{ }^{1}$ Laboratory of Structural Molecular Pharmacology, Graduate School of Pharmaceutical

10 Sciences, Nagoya University, Furocho, Chikusa-ku, Nagoya 464-8601, Japan

$11{ }^{2}$ BeCellBar LLC, Business Incubation Building, Nagoya University, Furocho, Chikusa-ku,

12 Nagoya, Aichi, 464-8601, Japan

14 Corresponding Author:

15 Hidekazu Hiroaki ${ }^{1,2}$

16 Laboratory of Structural Molecular Pharmacology, Graduate School of Pharmaceutical Sciences,

17 Nagoya University, Furocho, Chikusa-ku, Nagoya 464-8601, Japan

18 Email address: hiroaki.hidekazu@f.mbox.nagoya-u.ac.jp 


\section{Abstract}

22 Background. Intrinsically disordered proteins (IDPs) have been shown to exhibit cryoprotective 23 activity toward other cellular enzymes without any obvious conserved sequence motifs. This 24 study investigated relationships between the physical properties of several human genome25 derived IDPs and their cryoprotective activities.

26

27

28

29

30

31

32

33

34

35

36

37

38

39

40

41

42

43

44

45

46

47

48

49

50

51

52

53

54

55

56

57

58

59

Methods. Cryoprotective activity of three human-genome derived IDPs and their truncated peptides toward lactate dehydrogenase (LDH) and glutathione S-transferase (GST) was examined. After the shortest cryoprotective peptide was defined (named FK20), cryoprotective activity of all-D-enantiomeric isoform of FK20 (FK20-D) as well as a racemic mixture of FK20 and FK20-D was examined. In order to examine the lack of increase of thermal stability of the target enzyme, the CD spectra of GST and LDH in the presence of a racemic mixture of FK20 and FK20-D at varying temperatures were measured and used to estimate $T_{m}$.

Results. Cryoprotective activity of IDPs longer than 20 amino acids was nearly independent of the amino acid length. The shortest IDP-derived 20 amino acid length peptide with sufficient cryoprotective activity was developed from a series of TNFRSF11B fragments (named FK20) was identified. FK20, FK20-D, and an equimolar mixture of FK20 and FK20-D also showed similar cryoprotective activity toward LDH and GST. $\mathrm{T}_{\mathrm{m}}$ of GST in the presence and absence of an equimolar mixture of FK20 and FK20-D are similar, suggesting that IDPs' cryoprotection mechanism stems partly from a molecular shielding effect rather than a direct interaction with the target enzymes.

\section{Introduction}

Intrinsically disordered proteins (IDPs) are an important class of proteins, that is widely associated with broad biological processes (Wright \& Dyson, 1999; Romero et al., 2001; Dunker et al., 2008; Tompa \& Fersht, 2009; Uversky \& Dunker, 2010). The most unique feature of IDPs is that they lack stable and compact tertiary structures alone under physiological conditions, while some of them eventually fold into stable structures during specific interaction. Currently, at least two major biological functions of IDPs have been proposed: "coupling folding and binding" (Dyson \& Wright, 2002; Sugase, Dyson \& Wright, 2007; Higo, Nishimura \& Nakamura, 2011) and "accelerated association with partner molecules by the fly-casting mechanism" (Levy, Onuchic \& Wolynes, 2007; Sugase, Dyson \& Wright, 2007; Chen, 2009). The former function is amino acid sequence dependent, because at least some part of the IDP must adopt into a fixed conformation with specific molecular contacts upon target binding. The latter function is assumed as less sequence dependent; a higher flexibility in the extended conformation of the IDP region that interconnects two functional domains, is seemingly more important. Recently, we and other researchers proposed a third physiological function of IDPs as protectants from environmental stresses, such as freezing and desiccation (Hughes \& Graether, 
60

61

62

63

64

65

66

67

68

69

70

71

72

73

74

75

76

77

78

79

80

81

82

83

84

85

86

87

88

89

90

91

92

93

94

95

96

97

98

99

2011; Hughes et al., 2013; Boothby et al., 2017; Matsuo et al., 2018). In this study, we focused on the cryoprotective activity of IDPs.

Plant dehydrins (DHNs) are extensively well studied examples of cryoprotective IDPs (Allagulova et al., 2003; Hanin et al., 2011; Graether \& Boddington, 2014). DHNs belong to a family of late embryogenesis abundant (LEA) proteins that are major contributors to the development of desiccation tolerance during plant seed maturation (Goyal, Walton \& Tunnacliffe, 2005; Hincha \& Thalhammer, 2012; Amara et al., 2014). DHNs are characterized by the presence of one or more uniquely conserved sequence motifs, Lys-rich (K-), Tyr-rich (Y), and Ser-rich (S-) segments (Hughes \& Graether, 2011). Many in vitro studies have demonstrated that DHNs effectively prevent inactivation of the model reporter enzyme, lactate dehydrogenase (LDH), during repeated freeze/thaw cycles (Hughes \& Graether, 2011; CuevasVelazquez, Rendon-Luna \& Covarrubias, 2014). A possible mechanism underlying DHN cryoprotection involves "molecular shields" that prevent stochastic direct contacts between enzyme molecules (Chakrabortee et al., 2012; Hughes et al., 2013). Two independent research groups found that the cryoprotective activity of DHNs and their artificial variants roughly correlated with their hydrodynamic radius $\left(\mathrm{R}_{\mathrm{H}}\right)$ rather than their amino acid sequences (Hughes et al., 2013; Cuevas-Velazquez, Rendon-Luna \& Covarrubias, 2014; Ferreira et al., 2018), partly supporting the molecular shielding hypothesis.

Strongly encouraged by Graether and colleagues' work (Hughes \& Graether, 2011; Hughes et al., 2013; Ferreira et al., 2018), we have uncovered evidence supporting the molecular shielding hypothesis of cryoprotective IDPs through a different approach (Matsuo et al., 2018). If the cryoprotective action is driven solely by the molecular shielding effect, any other IDPs could also exert cryoprotective activity. Indeed, we found a potential example in the literature, silk worm sericin, a Ser-rich IDP (Tsujimoto et al., 2001). We expanded the cryoprotective IDP concept to include other evolutionarily unrelated IDPs, such as human genome-derived IDPs of 36 to 44 amino acid residues, rather than DHNs or LEAs. In our previous study (Matsuo et al., 2018), we demonstrated that all the examined IDPs derived from the human genome exerted cryoprotective activity toward not only the model enzyme LDH, but also glutathione-S transferase (GST) and green fluorescent protein (GFP). In detail, 53 candidate IDP genes from human genome were first predicted by a bioinformatics method, and then, among them, 35 IDP peptides systematically proven as the flexible disordered peptide segments by the NMR-based indirect IDP-assessment methods developed by us (Goda et al., 2015b). Accordingly, the five randomly-selected human genome-derived IDP peptides among the 35 peptides were demonstrated to have substantial cryoprotective activities against LDH, GST, and green fluorescent protein (Matsuo et al., 2018). However, in that study, all five IDPs showed similar levels of cryoprotective activity; we could not identify further sequence-activity relationships in the human genome-derived IDPs.

In this study, we selected three IDPs, C1, D10, and E1, for further analysis. We succeeded in minimizing the length of the human-genome-derived IDPs with practical cryoprotective activity. We found that FK20 - the 20 amino acid fragment (position 24-43) from 
100

101

102

103

104

105

106

107

108

109

110

111

112

113

114

115

116

117

118

119

120

121

122

123

124

125

126

127

128

129

130

131

132

133

134

135

136

137

138

the tumor necrosis factor receptor superfamily member 11B (TNFRS11B) precursor-showed substantial cryoprotective activity toward LDH. Then we compared the cryoprotective activity of FK20 and its all-D-enantiomeric isomer and found that the cryoprotective activity of FK20 is independent to its chirality. Accordingly, we employed a new technique to use the racemic mixture of FK20 and FK20-D, that do not show CD signal, to investigate the absence of specific molecular effect toward the GST and LDH reporter enzyme by CD spectroscopy. These results suggest a potential use of human genome-derived IDP as a cryopreserving agent for cryosensitive enzymes and proteins.

\section{Materials \& Methods}

\section{Expression and preparation of the IDP samples}

Human genome-derived IDP samples and their truncation mutants were prepared by an E. coli expression system optimized for preparing IDP samples, as previously described (Goda et al., 2015a). In brief, the N-terminal autoprotease N(pro) from bovine viral diarrhea virus was selected as a fusion partner for protein expression using the pET-based $\mathrm{N}$ (pro) fusion protein expression system (Achmüller et al., 2007). The IDPs were expressed, N-terminal tags were removed, and finally purified by reversed phase HPLC (COSMOSIL $₫$ 5C4-AR-300, Nacalai Tesque, $\phi 4.6 \mathrm{~mm} \times 250 \mathrm{~mm}$ ) with $0.1 \%$ trifluoroacetic acid-acetonitrile solvent system. All the peptides were quantified by UV absorbance at $280 \mathrm{~nm}$, lyophilized, and stored at $-30^{\circ} \mathrm{C}$ until use. FK20, a 20 amino acid peptide, and its all-D-enantiomeric isomer, FK20-D, were chemically synthesized (Biologica Co. Ltd. Nagoya, Aichi, Japan) with at least $80 \%$ purity, and purified by reversed phase HPLC.

\section{Cryoprotection assay for LDH}

Rabbit muscle lactate dehydrogenase (LDH) (Sigma-Aldrich, L-2500) was selected as the reporter enzyme for cryoprotection activity of shorter IDP peptides, using a slightly modified protocol based on Hughes and Graether (Hughes \& Graether, 2011). The initial LDH solution contained $50 \mu \mathrm{g} / \mathrm{ml}$ in $10 \mathrm{mM}$ sodium phosphate ( $\mathrm{pH}$ 7.4). In a 1.5-ml microfuge tube, we mixed a $10-\mu 1$ aliquot of the LDH solution with $10 \mu \mathrm{l}$ of solution containing each of the individual protectants (IDP peptides) at concentrations ranging from $2.5 \mu \mathrm{g} / \mathrm{ml}$ to $500 \mu \mathrm{g} / \mathrm{ml}$. Five cycles of freezing in liquid nitrogen for $30 \mathrm{~s}$ and thawing in a water bath at $4{ }^{\circ} \mathrm{C}$ for $5 \mathrm{~min}$ were applied to each sample. Subsequently, LDH activity was measured using a standard NADH oxidase coupled-enzyme system according to the our previous report (Matsuo et al., 2018). For the analysis, we set the LDH activity of the untreated sample (the enzyme without freeze and thaw processes and without the addition of a cryoprotectant) as $100 \%$. All measurements were performed in triplicate.

\section{Cryoprotection assay for GSH}


139 Glutathione S-transferase from Schistosoma japonicum (GST) was selected as the second

140 reporter enzyme for cryoprotection activity of shorter IDP peptides because this enzyme was

141 easily prepared in the laboratory and also suited for the subsequent CD spectroscopy

142 experiments. The cryoprotection assay was performed according to our previous report (Matsuo

143 et al., 2018). After five freeze/thaw cycles, GST activity was measured using a standard 1-

144 chloro-2,4-dinitrobenzene (CDNB, Sigma-Aldrich, 138630) assay with a 96-well plate and 2300

145 EnSpire Microplate Reader (Perkin Elmer). For the analysis, we set the GST activity of the

146 untreated sample (the enzyme without freeze and thaw processes and without the addition of a

147 cryoprotectant) as $100 \%$. All measurements were performed three times and the standard

148 deviations were calculated.

149

150

151

152

153

154

155

156

157

158

159

160

161

162

163

164

165

166

167

168

\section{Circular dichroism (CD) measurements}

CD spectra between 190 and $300 \mathrm{~nm}$ were collected on a J-805 spectropolarimeter (JASCO, Tokyo, Japan). The time constant, scan speed, bandwidth/resolution, and sensitivity of the spectropolarimeter were set at $1 \mathrm{~s}, 100 \mathrm{~nm} / \mathrm{min}, 1 \mathrm{~nm}$, and $100 \mathrm{mdeg}$, respectively. We measured a $300 \mu 1$ solution of $250 \mu \mathrm{g} / \mathrm{ml}$ of FK20, FK20-D or $125 \mu \mathrm{g} / \mathrm{ml}$ each of the FK20/FK20-D mixture with a $10 \mathrm{mM}$ sodium phosphate buffer $(\mathrm{pH} 7.4)$ in a quartz cuvette with a $1 \mathrm{~mm}$ light path length at $20^{\circ} \mathrm{C}$. Accordingly, we measured CD spectra of a $300 \mu \mathrm{l}$ solution of $100 \mu \mathrm{g} / \mathrm{ml}$ of GST with 0 , 25,50 and $100 \mu \mathrm{g} / \mathrm{ml}$ each of FK20/FK20-D mixture in the same buffer. Similarly, we measured CD spectra of a $300 \mu \mathrm{l}$ solution of $100 \mu \mathrm{g} / \mathrm{ml}$ of LDH with $0,25,50$ and $100 \mu \mathrm{g} / \mathrm{ml}$ each of FK20/FK20-D mixture in the same buffer.

The GST and LDH denaturation temperatures $\left(\mathrm{T}_{\mathrm{m}}\right)$ were determined by CD spectra at varying temperatures from $25^{\circ} \mathrm{C}$ to $75^{\circ} \mathrm{C}$ or $85^{\circ} \mathrm{C}$ by monitoring the ellipticity at $222 \mathrm{~nm}$ at the speed of heating by $1^{\circ} \mathrm{C} / \mathrm{min}$. Every $10^{\circ} \mathrm{C}, \mathrm{CD}$ spectra between 200 and $300 \mathrm{~nm}$ were automatically collected. For $\mathrm{LDH}, \mathrm{CD}$ spectra of $\mathrm{LDH}$ were measured at $25^{\circ} \mathrm{C}$ and $85^{\circ} \mathrm{C}$. A moving average of $\mathrm{CD}$ values of each five temperature points were plotted, and three straight lines corresponding to the baseline, the plateau, and the slope, were indicated. $\mathrm{T}_{\mathrm{m}}$ was determined as the temperature of $50 \%$ denatured state.

Results

\section{Cryoprotective activity of shorter IDPs toward LDH and GST}

170

To determine the minimal length of IDPs showing a substantial cryoprotective activity toward LDH and GST, we constructed several recombinant plasmids containing coding regions of the human genome-derived IDPs C1, D10, E1, and a series of their C-terminal truncated mutants. Amino acid sequences with schematic diagrams of IDPs and their truncated mutants are shown in Figure 1. Human genome-derived IDPs C1, D10, and E1 consisted of 36, 37 and 39 amino acids, respectively. These peptides were proven as IDPs by both $\mathrm{CD}$ spectra and ${ }^{1} \mathrm{H}-{ }^{15} \mathrm{~N} 2 \mathrm{D}-$ NMR spectra in our previous studies (Goda et al., 2015b; Matsuo et al., 2018). We used the PONDR server (http://pondr.com) (Obradovic et al., 2005) to predict whether the series of Cterminal truncated peptides were also disordered. Accordingly, the charge-hydropathy plots (Uversky plots) were shown in Supplementary Figure S1. Cryoprotective activity of all peptides 
180

181

182

183

184

185

186

187

188

189

190

191

192

193

194

195

196

197

198

199

200

201

202

203

204

205

206

207

208

209

210

211

212

213

214

215

216

217

218

219

toward LDH at concentrations of 5, 10, 25, 50 and $500 \mu \mathrm{g} / \mathrm{mL}$ were measured. All peptides showed stronger cryoprotective activity than BSA in a concentration-dependent manner, with none showing less than $90 \%$ cryoprotection at the highest concentration $(500 \mu \mathrm{g} / \mathrm{mL}$ ) (data not shown). We compared these cryoprotective effects at the intermediate concentration $(50 \mu \mathrm{g} / \mathrm{mL})$ (Figure 2A) and found that the cryoprotective activity of most of IDPs was independent of their amino acid lengths, except the three shorter peptides, $\mathrm{C}^{20}, \mathrm{D} 10^{15}$ and $\mathrm{E} 1^{20}$. These peptides showed decreased cryoprotection activity of less than $60 \%$. As a result, D $10^{20}$ was the shortest peptide with practical cryoprotective activity toward LDH at $50 \mu \mathrm{g} / \mathrm{mL}$.

Subsequently, we examined the cryoprotective activity of these peptides toward GST (Figure 2B) and the results were similar to those found with LDH. Also, D10 ${ }^{20}$ was again the shortest peptide with reasonably sufficient cryoprotective activity toward GSH at $50 \mu \mathrm{g} / \mathrm{mL}$. We further analyzed the normalized cryoprotective activity by amino acid lengths and calculated isoelectric points, pI (Figures $2 \mathrm{C}$ and 2D, respectively). In order to compare the cryoprotective activities for the different reporter enzymes, both the maximum preserved enzymatic activities with the cryoprotective peptide, LDH with $\mathrm{E}^{31}$ and GST with D10 ${ }^{20}$, were set to $100 \%$, respectively. Peptides shorter than 20 amino acids were less cryoprotective, which is consistent with the proposed cryoprotection mechanism of DHNs as a molecular shield effect, where cryoprotective activity of various DHNs were roughly proportional to $\mathrm{R}_{\mathrm{H}}$ logarithms of the cryoprotectants (Cuevas-Velazquez, Rendon-Luna \& Covarrubias, 2014; Ferreira et al., 2018). Since we only examined cryoprotective IDPs smaller than 42 residues, only the lower limit of the correlation has been observed. In addition, we found that the IDP peptides with either extremely high or low $\mathrm{pI}$ were less cryoprotective, whereas the peptides with neutral $\mathrm{pI}$ were more potent. Hereafter, our shortest cryoprotective IDP-derived peptide D10 ${ }^{20}$ is referred to as FK20.

\section{Cryoprotective activity of FK20 and its all-D-enantiomeric isomer FK20-D}

We further characterized cryoprotective activity and its FK20 mechanism by using its all-Denantiomeric isomer, FK20-D. Based on this study and our previous study, the cryoprotective activity of IDP-derived peptides is likely not amino acid sequence specific. For example, we measured and compared ${ }^{1} \mathrm{H}^{-15} \mathrm{~N}$ HSQC spectra of the target molecule $\mathrm{A} \beta(1-42)$ in the absence and the presence of the cryoprotective IDPs in our previous study, and we observed almost no spectra change (Ikeda et al., 2020). Thus, the absence of any specific molecular interaction between IDPs and the target enzymes is expected. It is generally saying that proving nonexistence is always difficult.Although it is difficult to completely prove the absence of any specific interaction between the IDPs and the reporter enzymes, we challenged to provide indirect evidence to support this assumption as many as possible. In this study, we examined the cryoprotective activity of FK20-D and compared to that of the parent FK20. The cryoprotective activities of FK20, FK20-D, and the same total concentration of the racemic equimolar mixture of FK20 and FK20-D (FK20-LD) toward LDH and GST at varying concentrations were examined (Figure 3A and 3B, respectively). FK20-D showed similar concentration-dependent cryoprotective profiles toward the reporter enzymes. The results suggested that specific

PeerJ Phy. Chem. reviewing PDF | (PCHEM-2020:12:56382:1:0:NEW 28 Oct 2021) 
220

221

222

223

224

225

226

227

228

229

230

231

232

233

234

235

236

237

238

239

240

241

242

243

244

245

246

247

248

249

250

251

252

253

254

255

256

257

258

molecular interaction between the reporter enzymes and either the FK20 or FK20-D peptides was absent, because the contribution of any residue-specific interaction of FK20 is not reproducible with FK20-D. We assumed that the effect of FK20 and FK20-D toward LDH and GST were more "environmental". Indeed, FK20-LD showed similar concentration-dependent cryoprotective profiles (Figures $3 \mathrm{~A}$ and $3 \mathrm{~B}$ ). Note that the additive property of different cryoprotective IDPs was also observed between full length C1 and D10 (Supplementary Figure S2). A 1:1 mixture (in weight) of C1 and D10 showed a concentration-dependent cryoprotective profile similar to that of $\mathrm{C} 1$.

\section{Effect of cryoprotective IDP peptide toward structure and thermal stability of GST and LDH}

Next we tried to show that the cryoprotective activity was not due to either structural change or thermal stabilization of GST, but rather that reporter enzyme cryoprotection can be explained by avoiding denaturation of the enzymes by structural or thermal stabilization through either specific or non-specific IDP interactions. In our the other previous study, we succeeded in demonstrating the amyloid formation inhibitory activity of the same human genome-derived IDP peptides against $A \beta(1-42)$ (Ikeda et al., 2020). At that time, we employed solution NMR techniques to monitor existence of a specific molecular interaction between ${ }^{15} \mathrm{~N}$-labelled $\mathrm{A} \beta(1-$ 42 ) in the presence of non-labelled IDPs. However, in this study, the molecular weight of the reporter enzyme (for example, GST) seems not suitable for solution NMR. Therefore, we employed CD spectroscopy, since FK20-LD, the equimolar mixture of FK20 and FK20-D, is silent in $\mathrm{CD}$ measurement. Figure 4A presents the experimental evidence of this unique feature of FK20-LD (grey), showing no significant CD band. FK-20 (black) showed the typical CD spectrum of a disordered state, whereas FK20-D (black, dashed line) showed the exact same CD spectrum with an inverted sign, as expected.

Figure 4B shows the CD spectra of GST in the absence (dashed line) and presence (solid line) of FK20-LD. There was no significant change in CD spectra, indicating that GST did not change its three-dimensional structure. Thus, FK20-LD cryoprotective activity was not a result of GST structural change. Figures 4C and 4E show a series of GST CD spectra at increasing temperatures from $25^{\circ} \mathrm{C}$ to $75^{\circ} \mathrm{C}$ with and without FK20-LD. Figure 4D and 4F show GST thermal denaturation plots taken from Figures $4 \mathrm{C}$ and $4 \mathrm{E}$, respectively. The GST thermal denaturation temperature $\left(\mathrm{T}_{\mathrm{m}}\right)$ was $56.5 \pm 1.2^{\circ} \mathrm{C}$ for GST alone, and $58.2 \pm 1.1^{\circ} \mathrm{C}$ in the presence of $0.2 \mathrm{mg} / \mathrm{mL}$ of FK20-DL. Similarly, Figure 5A shows the CD spectra of LDH in the absence (dashed line) and presence (solid line) of FK20-LD. Figures 5B and 5D show initial and final $\mathrm{LDH} C \mathrm{CD}$ spectra at increasing temperatures from $25^{\circ} \mathrm{C}$ to $75^{\circ} \mathrm{C}$ with and without FK20-LD. Figure 5C and 5E show $\mathrm{LDH}$ thermal denaturation plots taken from Figures 5B and 5D s, respectively. The $\mathrm{LDH}$ thermal denaturation temperature $\left(\mathrm{T}_{\mathrm{m}}\right)$ was $61.2^{\circ} \mathrm{C}$ for $\mathrm{LDH}$ alone, and $62.0^{\circ} \mathrm{C}$ in the presence of $0.2 \mathrm{mg} / \mathrm{mL}$ of FK20-DL. The results suggest that the thermal stability gains of GST and LDH by FK20-DL are small. Thus, we concluded that the FK20-DL 
259

260

261

\section{Discussion}

263

264

265

266

267

268

269

270

271

272

273

274

275

276

277

278

279

280

281

282

283

284

285

286

287

288

289

290

291

292

293

294

295

296

297 enzymes. enzymes.

cryoprotective activity was likely not a result of a thermal stabilization effect on the reporter

In this study, we succeeded in minimizing the amino acid length of human-genome-derived IDPs with a reasonable cryoprotective effect into 20 amino acid residues. The shortest cryoprotective peptide, FK20, showed $100 \%$ cryoprotective action toward LDH at a concentration of 100 $\mu \mathrm{g} / \mathrm{mL}(0.01 \%)$. We observed that the peptides shorter than 20 amino acids were less cryoprotective. The result is consistent with the proposed cryoprotection mechanism of DHNs as a molecular shield effect, where cryoprotective activity of various DHNs were roughly proportional to hydrodynamic radius $\mathrm{R}_{\mathrm{H}}$ logarithms of the cryoprotectants (Cuevas-Velazquez, Rendon-Luna \& Covarrubias, 2014; Ferreira et al., 2018).The mechanism of molecular shield is to inhibit a core of protein aggregation during freeze-thaw cycles of the proteins by disturbing the direct collision between the reporter enzymes. Thus, $\mathrm{R}_{\mathrm{H}}$ of cryoprotectant is considered for explaining the molecular shield efficacy.

We demonstrated that the cryoprotective activity of FK20 is independent to its molecular chirality, that suggests the absence of the specific interaction between the reporter enzymes and FK20. This assumption is consistent with our observation that the addition of IDP (in this time, FK20 / FK20-D mixture) did not affect the thermal stability of GST and LDH. The GST thermal denaturation temperature $\left(\mathrm{T}_{\mathrm{m}}\right)$ was $56.6 \pm 1.1^{\circ} \mathrm{C}$ for GST alone, and $58.1 \pm 1.1^{\circ} \mathrm{C}$ in the presence of $0.2 \mathrm{mg} / \mathrm{mL}$ of FK20-DL (Figure 4D and 4F). Similarly, the $\mathrm{T}_{\mathrm{m}}$ of LDH was $61.2^{\circ} \mathrm{C}$ for LDH alone, and $62.0^{\circ} \mathrm{C}$ in the presence of $0.2 \mathrm{mg} / \mathrm{mL}$ of FK20-DL, and the remarkable gain of thermal stability was not observed (Figure 5C and 5E). Thus, we concluded that the FK20-DL cryoprotective activity was likely not a result of a thermal stabilization effect on the reporter

The FK20 mechanism of cryoprotective activity toward the reporter enzymes seems to be a molecular shield effect, in which the peptide may prevent stochastic contacts with the reporter enzyme molecules upon desiccation during the freezing process. The schematic diagrams of cryoprotecting mechanisms toward the reporter enzymes are summarized in Figures 6. Recently, a new class of functional IDPs that exhibits a potent protective activity against protein aggregation following to heat-denaturation, named heat-resistant obscure (Hero) proteins was discovered (Tsuboyama et al., 2020). Although the mechanism of aggregation protection of Hero proteins is not yet unraveled, the molecular shield mechanism is one of the candidates. We recently reported that human genome-derived IDPs also can inhibit the nucleation phase of the amyloid fibril formation of $A \beta(1-42)$ (Ikeda et al., 2020), and the fibril formation inhibition is likely based on the molecular shield mechanism.

In conclusion, we have developed a cryoprotective peptide, FK20, with 20 amino acid residues. The sequence of FK 20 was taken from human genome encoded intrinsically disordered proteins. The mechanism of cryoprotection toward the reporter enzymes, LDH and GST, was

Peer] Phy. Chem. reviewing PDF | (PCHEM-2020:12:56382:1:0:NEW 28 Oct 2021) 
298

299

300

301

302

303

304

305

306

307

308

309

310

311

312

313

314

315

316

317

318

319

320

321

322

323

324

325

326

327

328

329

330

331

332

333

334

335

336

337

338

339

340

341

assumed to be a molecular shield effect with no specific molecular interaction. Taking into consideration of these facts, the molecular shield becomes one of noteworthy properties of IDPs.

\section{Conclusions}

We have developed a cryoprotective peptide, FK20, with 20 amino acid residues. The sequence of FK20 was taken from human genome encoded intrinsically disordered proteins. FK20, its allD-enantiomeric isomer FK20-D, and the equimolar mixture of FK20 / FK20-D exhibited the similar level of cryoprotective activity toward the reporter enzymes, LDH and GST. No marked thermal stabilization of GST was observed in the presence of the equimolar mixture of FK20 / FK20-D. The mechanism of cryoprotection toward the reporter enzymes, LDH and GST, was assumed to be a molecular shield effect with no (stereo-)specific molecular interaction.

\section{References}

Achmüller C, Kaar W, Ahrer K, Wechner P, Hahn R, Werther F, Schmidinger H, CserjanPuschmann M, Clementschitsch F, Striedner G, Bayer K, Jungbauer A, Auer B. 2007. $\mathrm{N}$ (pro) fusion technology to produce proteins with authentic $\mathrm{N}$ termini in E. coli. Nature methods 4:1037-1043. DOI: 10.1038/nmeth1116.

Allagulova CR, Gimalov FR, Shakirova FM, Vakhitov VA. 2003. The Plant Dehydrins: Structure and Putative Functions. Biochemistry (Moscow). DOI: 10.1023/A:1026077825584.

Amara I, Zaidi I, Masmoudi K, Dolors Ludevid M, Pagès M, Goday A, Brini F. 2014. Insights into Late Embryogenesis Abundant (LEA) Proteins in Plants: From Structure to the Functions. American Journal of Plant Sciences. DOI: 10.4236/ajps.2014.522360.

Boothby TC, Tapia H, Brozena AH, Piszkiewicz S, Smith AE, Giovannini I, Rebecchi L, Pielak GJ, Koshland D, Goldstein B. 2017. Tardigrades Use Intrinsically Disordered Proteins to Survive Desiccation. Molecular Cell 65:975-984.e5. DOI: 10.1016/j.molcel.2017.02.018.

Chakrabortee S, Tripathi R, Watson M, Schierle GSK, Kurniawan DP, Kaminski CF, Wise MJ, Tunnacliffe A. 2012. Intrinsically disordered proteins as molecular shields. Molecular bioSystems 8:210-9. DOI: 10.1039/c1 mb05263b.

Chen J. 2009. Intrinsically disordered p53 extreme C-terminus binds to $\mathrm{S} 100 \mathrm{~B}(\beta \beta)$ through "flycasting." Journal of the American Chemical Society. DOI: 10.1021/ja809547p.

Cuevas-Velazquez CL, Rendon-Luna DF, Covarrubias AA. 2014. Dissecting the cryoprotection mechanisms for dehydrins. Frontiers in Plant Science 5. DOI: 10.3389/fpls.2014.00583.

Dunker AK, Silman I, Uversky VN, Sussman JL. 2008. Function and structure of inherently disordered proteins. Current opinion in structural biology 18:756-64. DOI: 10.1016/j.sbi.2008.10.002.

Dyson HJ, Wright PE. 2002. Coupling of folding and binding for unstructured proteins. Current Opinion in Structural Biology 12:54-60. DOI: 10.1016/S0959-440X(02)00289-0.

Ferreira LA, Walczyk Mooradally A, Zaslavsky B, Uversky VN, Graether SP. 2018. Effect of an Intrinsically Disordered Plant Stress Protein on the Properties of Water. Biophysical Journal. DOI: 10.1016/j.bpj.2018.09.014.

Goda N, Matsuo N, Tenno T, Ishino S, Ishino Y, Fukuchi S, Ota M, Hiroaki H. 2015a. An optimized N(pro)-based method for the expression and purification of intrinsically

Peer] Phy. Chem. reviewing PDF | (PCHEM-2020:12:56382:1:0:NEW 28 Oct 2021) 
disordered proteins for an NMR study. Intrinsically disordered proteins 3:e1011004. DOI: 10.1080/21690707.2015.1011004.

Goda N, Shimizu K, Kuwahara Y, Tenno T, Noguchi T, Ikegami T, Ota M, Hiroaki H. 2015b. A Method for Systematic Assessment of Intrinsically Disordered Protein Regions by NMR. International journal of molecular sciences 16:15743-60. DOI: 10.3390/ijms160715743.

Goyal K, Walton LJJ, Tunnacliffe A. 2005. LEA proteins prevent protein aggregation due to water stress. Biochemical Journal 388:151-157. DOI: 10.1042/BJ20041931.

Graether SP, Boddington KF. 2014. Disorder and function: a review of the dehydrin protein family. Frontiers in Plant Science. DOI: 10.3389/fpls.2014.00576.

Hanin M, Brini F, Ebel C, Toda Y, Takeda S, Masmoudi K. 2011. Plant dehydrins and stress tolerance: Versatile proteins for complex mechanisms. Plant Signaling and Behavior. DOI: 10.4161/psb.6.10.17088.

Higo J, Nishimura Y, Nakamura H. 2011. A free-energy landscape for coupled folding and binding of an intrinsically disordered protein in explicit solvent from detailed all-atom computations. Journal of the American Chemical Society 133:10448-10458. DOI: 10.1021/ja110338e.

Hincha DK, Thalhammer A. 2012. LEA proteins: IDPs with versatile functions in cellular dehydration tolerance: Figure 1. Biochemical Society Transactions. DOI: $10.1042 /$ bst20120109.

Hughes S, Graether SP. 2011. Cryoprotective mechanism of a small intrinsically disordered dehydrin protein. Protein Science 20:42-50. DOI: 10.1002/pro.534.

Hughes SL, Schart V, Malcolmson J, Hogarth K a, Martynowicz DM, Tralman-Baker E, Patel SN, Graether SP. 2013. The importance of size and disorder in the cryoprotective effects of dehydrins. Plant physiology 163:1376-86. DOI: 10.1104/pp.113.226803.

Ikeda K, Suzuki S, Shigemitsu Y, Tenno T, Goda N, Oshima A, Hiroaki H. 2020. Presence of intrinsically disordered proteins can inhibit the nucleation phase of amyloid fibril formation of $A \beta(1-42)$ in amino acid sequence independent manner. Scientific reports 10:12334. DOI: 10.1038/s41598-020-69129-1.

Levy Y, Onuchic JN, Wolynes PG. 2007. Fly-casting in protein-DNA binding: frustration between protein folding and electrostatics facilitates target recognition. Journal of the American Chemical Society 129:738-739.

Matsuo N, Goda N, Shimizu K, Fukuchi S, Ota M, Hiroaki H. 2018. Discovery of Cryoprotective Activity in Human Genome-Derived Intrinsically Disordered Proteins. International journal of molecular sciences 19:401. DOI: 10.3390/ijms19020401.

Obradovic Z, Peng K, Vucetic S, Radivojac P, Dunker AK. 2005. Exploiting heterogeneous sequence properties improves prediction of protein disorder. In: Proteins: Structure, Function and Genetics. 176-182. DOI: 10.1002/prot.20735.

Romero P, Obradovic Z, Li X, Garner EC, Brown CJ, Dunker AK. 2001. Sequence complexity of disordered protein. Proteins 42:38-48.

Sugase K, Dyson HJ, Wright PE. 2007. Mechanism of coupled folding and binding of an intrinsically disordered protein. Nature 447:1021-5. DOI: $10.1038 /$ nature05858.

Tompa P, Fersht A (eds.). 2009. Structure and Function of Intrinsically Disordered Proteins CRC Press Book. Chapman and Hall/CRC.

Tsuboyama K, Osaki T, Matsuura-Suzuki E, Kozuka-Hata H, Okada Y, Oyama M, Ikeuchi Y, Iwasaki S, Tomari Y. 2020. A widespread family of heat-resistant obscure (Hero) proteins 
387 protect against protein instability and aggregation. PLoS biology 18:e3000632. DOI:

$388 \quad$ 10.1371/journal.pbio.3000632.

389 Tsujimoto K, Takagi H, Takahashi M, Yamada H, Nakamori S, Sericin P. 2001. Cryoprotective

390 effect of the serine-rich repetitive sequence in silk protein sericin. Journal of biochemistry

391 129:979-986. DOI: 10.1093/oxfordjournals.jbchem.a002946.

392 Uversky VN, Dunker AK. 2010. Understanding protein non-folding. Biochimica et biophysica 393 acta 1804:1231-1264. DOI: 10.1016/j.bbapap.2010.01.017.

394 Wright PE, Dyson HJ. 1999. Intrinsically unstructured proteins: re-assessing the protein

395 structure-function paradigm. Journal of molecular biology 293:321-31. DOI:

$396 \quad 10.1006 /$ jmbi.1999.3110.

397

398 


\section{Figure 1}

Figure 1. Amino acid sequences and schematic diagrams of human genome-derived IDPs and their deletion mutants.

Fig. 1. Amino acid sequences and schematic diagrams of human genome-derived IDPs and their deletion mutants. Molecular weight (M.W.) and calculated isoelectric point (pl) are also shown. D10 $0^{20}$ was renamed FK20. Residues of D-amino acids are shown in italics. The Ref_seq accession codes, protein names, and the corresponding residue numbers of the human genome-derived IDPs are as follows; C1 (NP_570859.1 obsolete, WW domain-containing oxidoreductase isoform 3, 1-36), D10 (NP_002537.3, tumor necrosis factor receptor superfamily member 11B precursor, 24-62), and E1 (NP_005222.2, src substrate cortactin isoform a, 305-342). 


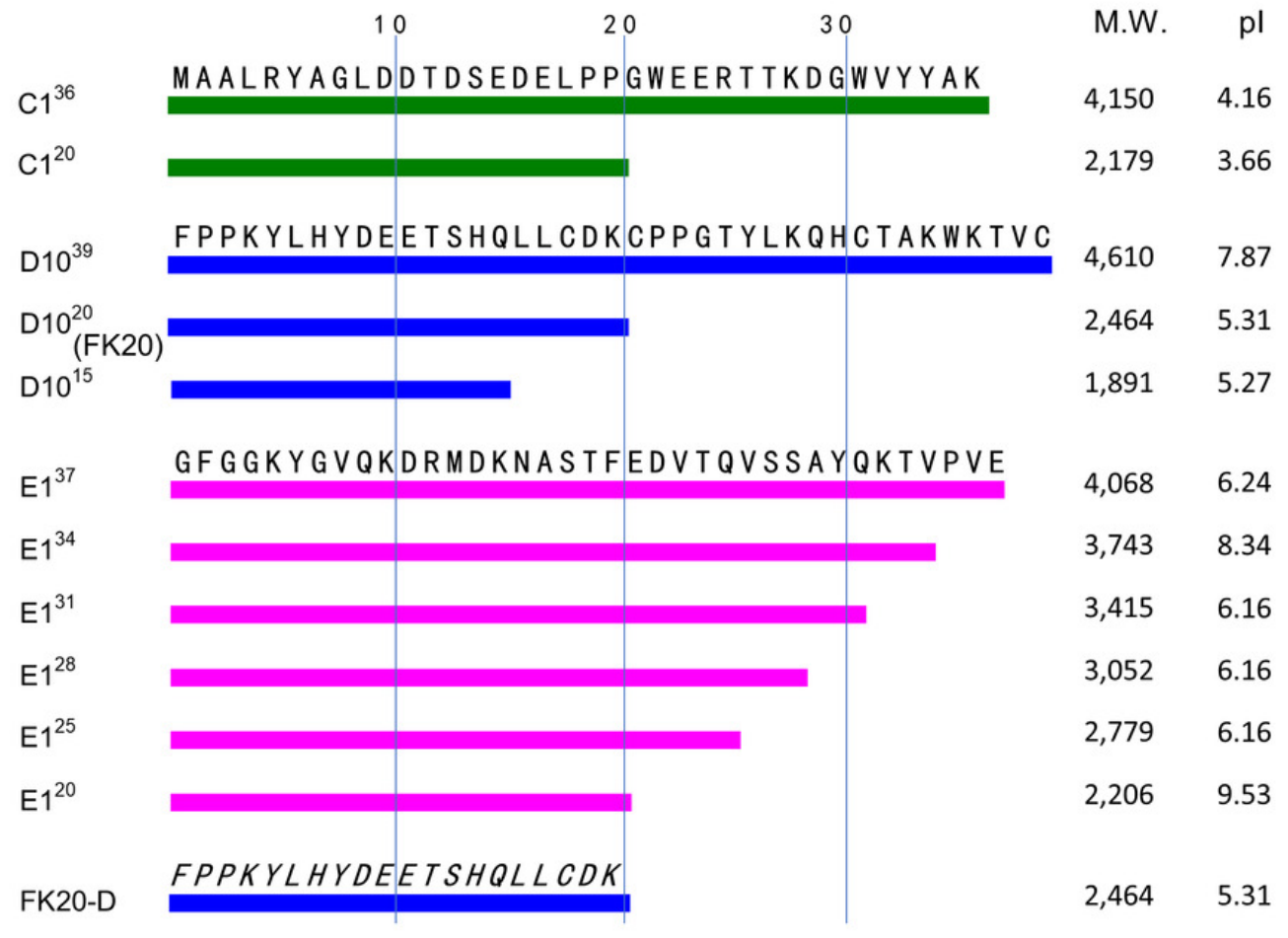




\section{Figure 2}

Figure 2. Cryoprotective activity of human genome derived IDPs against model enzymes LDH and GST.

Fig. 2. Cryoprotective activity of human genome-derived IDPs and control proteins towards LDH (A) and GST (B). Dependency of the normalized cryoprotective activity against amino acid lengths (C) and calculated isoelectric points, pl (D). (A) LDH activities (final concentration of $50 \mu \mathrm{g} / \mathrm{ml}$ ) after freeze-thawing in the presence of $50 \mu \mathrm{g} / \mathrm{ml}$ indicated additive IDP peptides. The LDH activity of the untreated sample was set to $100 \%$. Protein(-) indicates LDH activity without cryoprotectant. The error bars indicate standard deviation. (B) GST activities (final concentration of $280 \mu \mathrm{g} / \mathrm{ml}$ ) after freeze-thawing in the presence of 50 $\mu \mathrm{g} / \mathrm{ml}$ indicated additive IDP peptides. The GST activity of the untreated sample was set to 100\%. IDPs and peptides are colored black. Protein(-) indicates GST activity without cryoprotectant. The error bars indicate standard deviation. (C) Cryoprotective activities of each cryoprotectants towards LDH and GST are normalized and plotted against their amino acid length. (D) Normalized cryoprotective activities are plotted against pl of each cryoprotectants. 
Figure 2

A

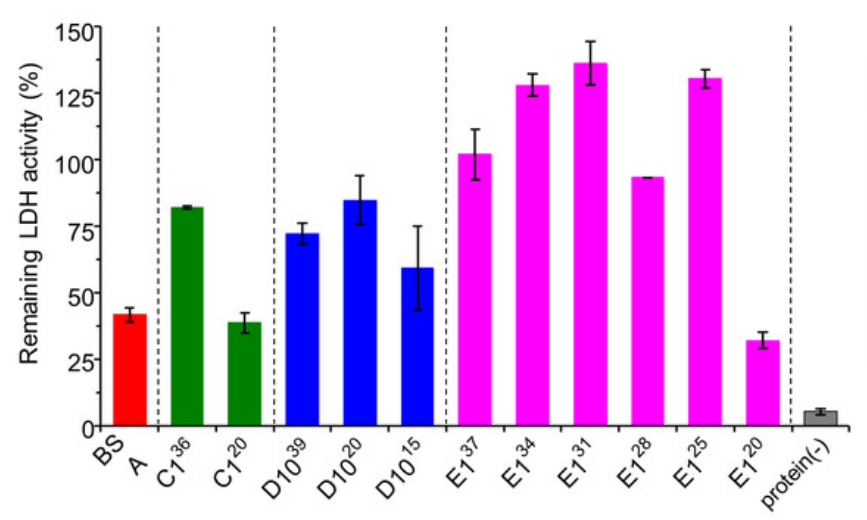

B

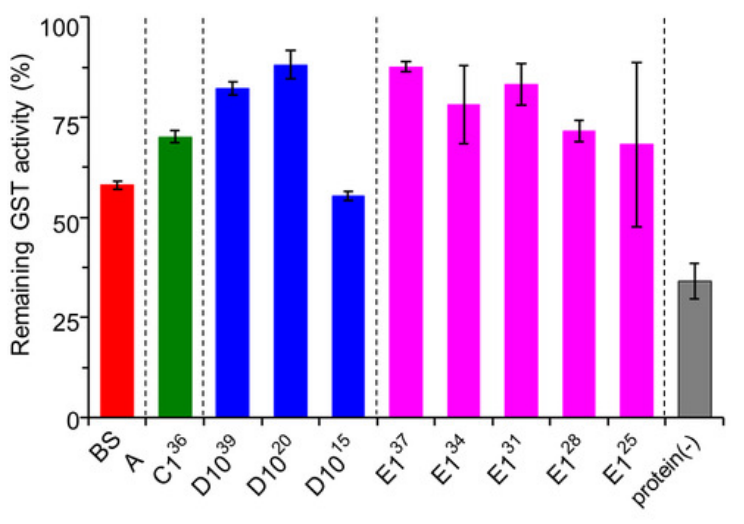

D

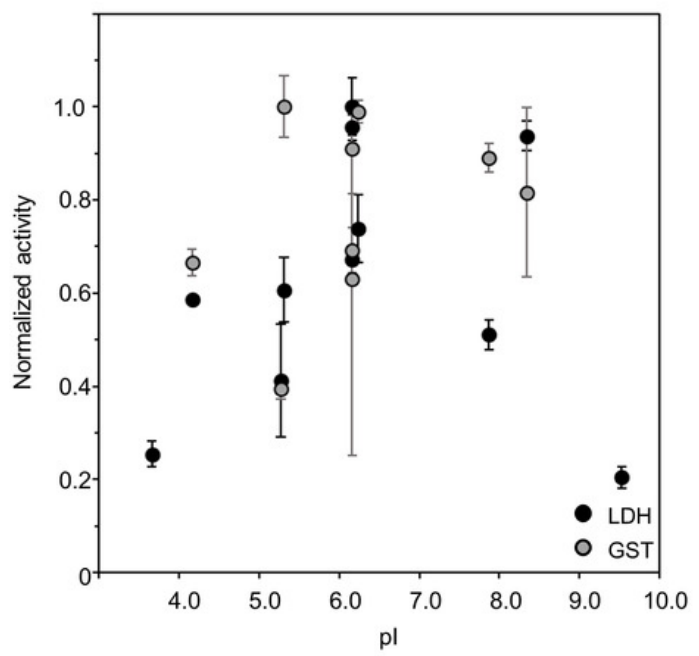

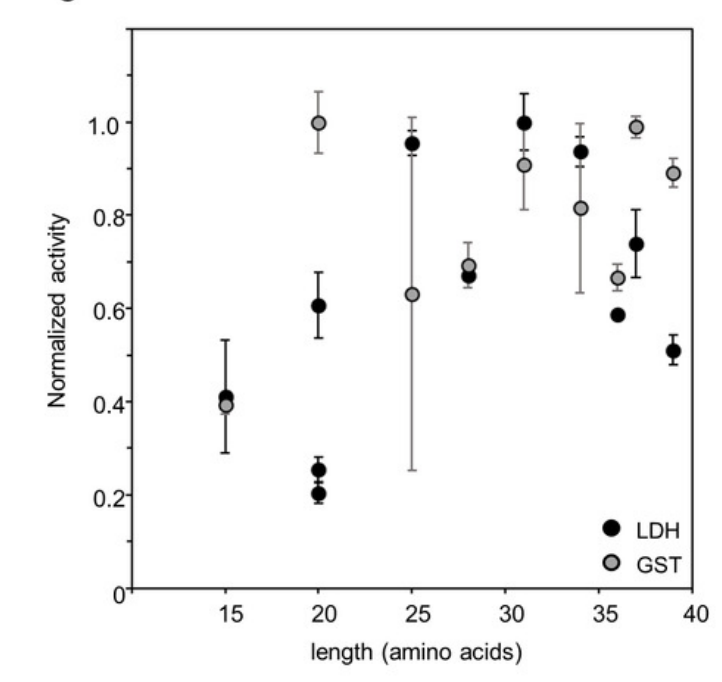

C

\footnotetext{
$\mathrm{pl}$
} 


\section{Figure 3}

Figure 3. Cryoprotective activity of IDP-derived peptide FK20 and its all D-enantiomeric isomer FK20-D towards LDH and GST.

Fig. 3. Cryoprotective activity of IDP-derived peptide FK20 and its all D-enantiomeric isomer FK20-D towards LDH (A) and GST (B) (A) LDH activities were plotted (final concentration of 50 $\mu \mathrm{g} / \mathrm{ml}$ ) after freeze-thawing in the presence of additive IDP peptides of concentrations ranging from 2.5 to $500 \mu \mathrm{g} / \mathrm{mL}$. The $\mathrm{LDH}$ activity of the untreated sample was set to $100 \%$. FK20: filled circle, FK20-D: grey square. (B) GST activities were plotted (final concentration of $280 \mu \mathrm{g} / \mathrm{mL}$ ) after freeze-thawing in the presence of additive IDP peptides of the concentration ranging 2.5 to $500 \mu \mathrm{g} / \mathrm{mL}$. The GST activity of the untreated sample was set to $100 \%$. FK20: filled circle, FK20-D; grey square, FK20-DL (equimolar mixture of FK20 and FK20-D): filled triangle with dashed line. 
Figure 3
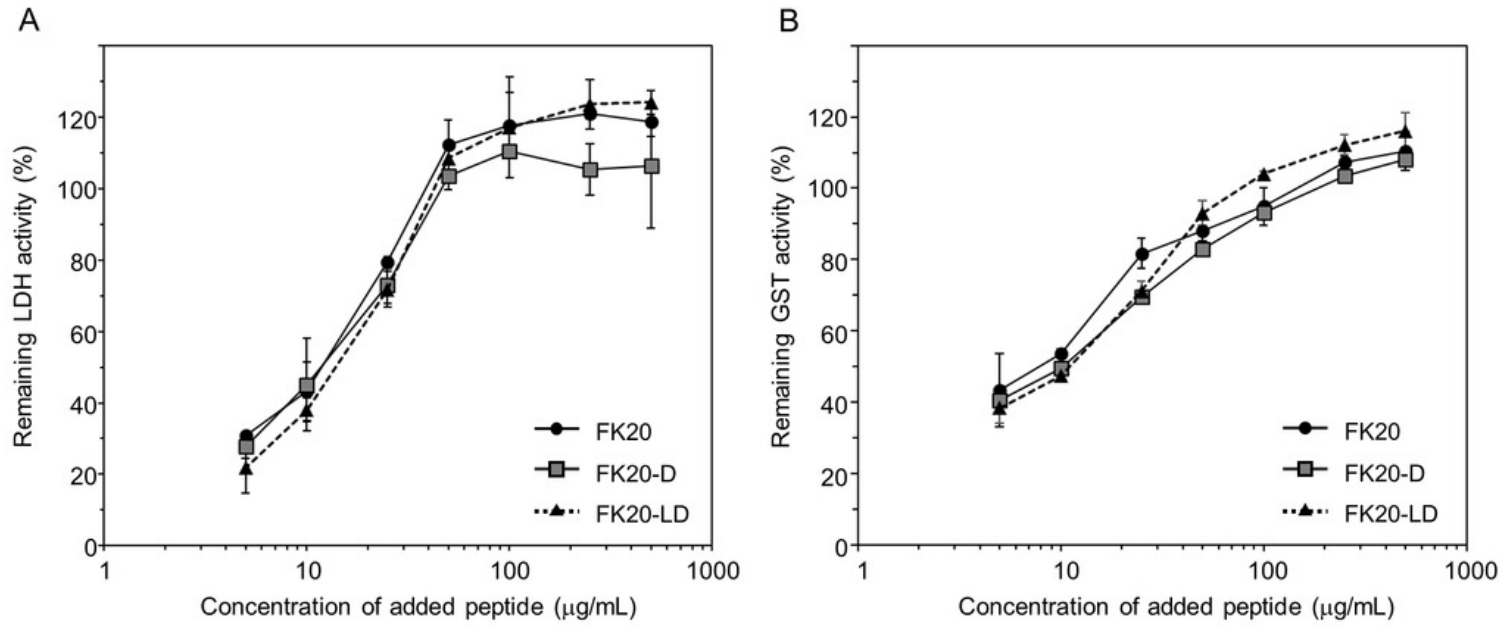


\section{Figure 4}

Figure 4. Influence of IDP-derived peptide FK20 and its all D-enantiomeric isomer FK20$D$ to the structure and thermal stability of GST.

Fig. 4. Influence of IDP-derived peptide FK20 and its all D-enantiomeric isomer FK20-D to the structure and thermal stability of GST. (A) CD spectra of $250 \mu \mathrm{g} / \mathrm{mL}$ of FK20 (solid line), FK20$\mathrm{D}$ (dashed line) and equimolar mixture (125 $\mathrm{\mu g} / \mathrm{mL}$ each) FK20-LD (grey line) at $25^{\circ} \mathrm{C}$. (B) CD spectra of $100 \mu \mathrm{g} / \mathrm{mL}$ GST without (dashed line) or with (solid lines) 50, 100, and $200 \mu \mathrm{g} / \mathrm{mL}$ of FK20-LD at $25^{\circ} \mathrm{C}$. (C) CD spectra of $100 \mu \mathrm{g} / \mathrm{mL}$ GST alone at various temperatures. (E) CD spectra of $100 \mu \mathrm{g} / \mathrm{mL}$ GST with $200 \mu \mathrm{g} / \mathrm{mL}$ of FK20-LD at various temperatures ranging 25 to $75^{\circ} \mathrm{C}$. (D, F) Melting curve of GST with (F) or without (D) FK20-LD monitored by the residual molar ellipticity [theta] at $222 \mathrm{~nm}$. 
Figure 4

A
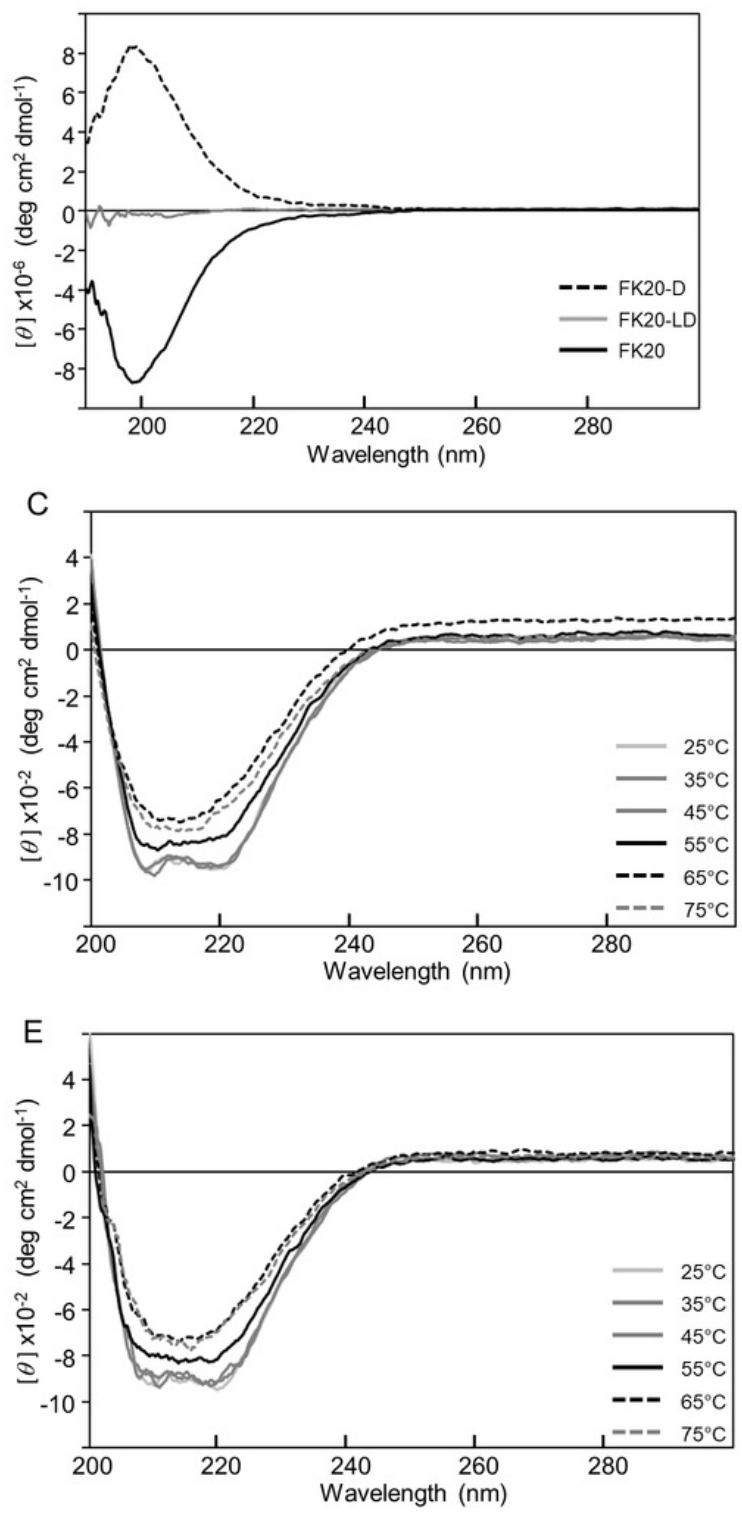

B
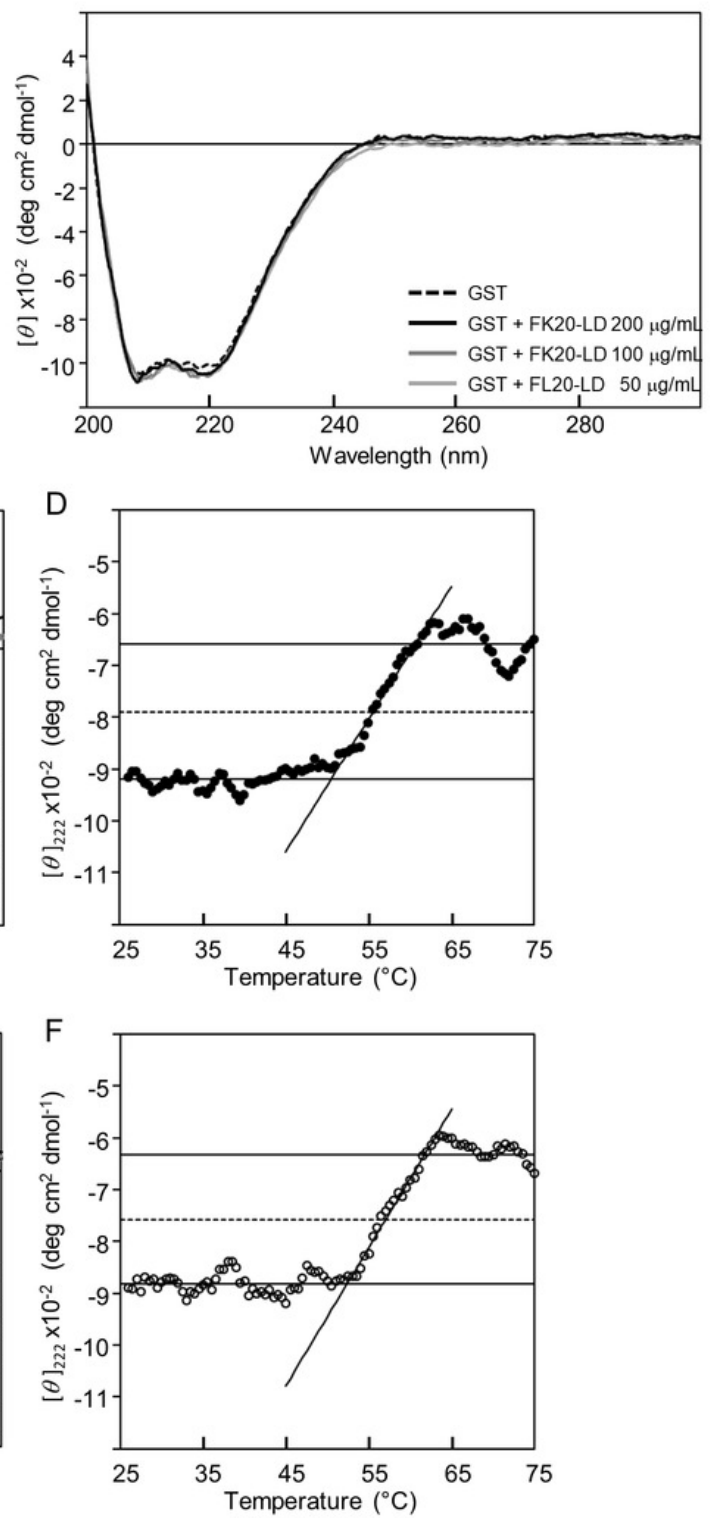


\section{Figure 5}

Figure. 5. Influence of IDP-derived peptide FK20 and its all D-enantiomeric isomer FK20$\mathrm{D}$ to the structure and thermal stability of LDH.

Fig. 5. Influence of IDP-derived peptide FK20 and its all D-enantiomeric isomer FK20-D to the structure and thermal stability of LDH. (A) CD spectra of $100 \mu \mathrm{g} / \mathrm{mL}$ LDH without (dashed line) or with (solid lines) 50,100 , and $200 \mu \mathrm{g} / \mathrm{mL}$ of FK20-LD at $25^{\circ} \mathrm{C}$. (B) CD spectra of 100 $\mu \mathrm{g} / \mathrm{mL} \mathrm{LDH}$ alone at two temperatures, 25 and $85^{\circ} \mathrm{C}$. (D) CD spectra of $100 \mu \mathrm{g} / \mathrm{mL} \mathrm{LDH}$ with $200 \mu \mathrm{g} / \mathrm{mL}$ of FK20-LD at two temperatures, 25 and $85^{\circ} \mathrm{C}$. (C, F) Melting curve of LDH with (E) or without (C) FK20-LD monitored by the residual molar ellipticity [theta] at $222 \mathrm{~nm}$. 


\section{Figure 5 new revised}
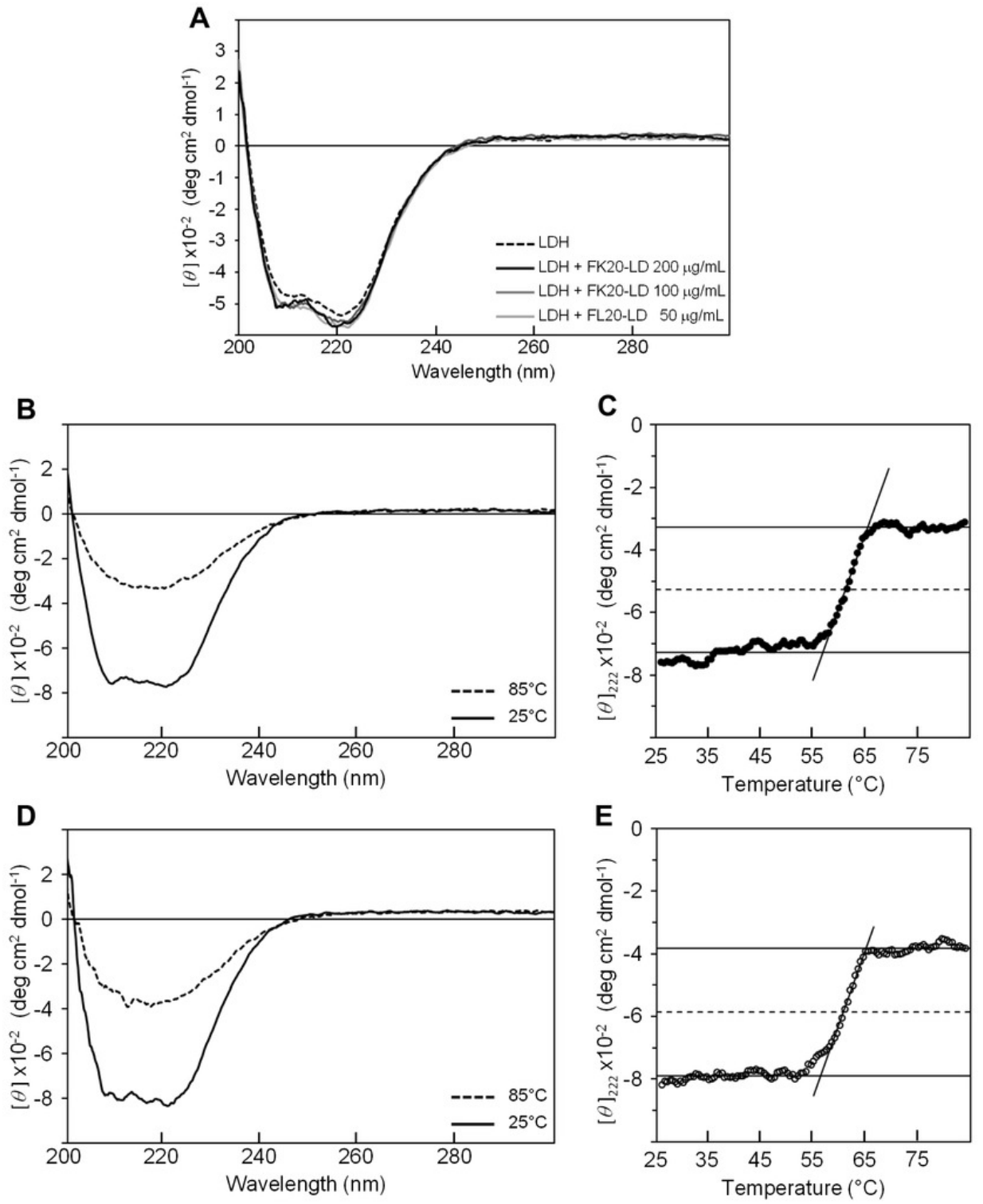


\section{Figure 6}

Figure. 6. Schematic representation of the mechanisms for cryoprotection of enzyme by FK20.

Fig. 6. Schematic representation of the mechanisms for cryoprotection of enzyme by FK20. (A) Illustration of the mechanism of stochastic protein collisions upon repeated freeze/thaw cycles. The GST molecule (dimer) is represented by the ribbon diagram. The filled block arrow indicates stochastic direct collision of proteins during freeze/thaw cycles. (B) Illustration of the molecular shield model of FK20 cryoprotective activity toward the enzymes, originally proposed by Chakrabortee et al. (see text) (Chakrabortee et al., 2012) . FK20 is represented as bold strings. 
Figure 6

A
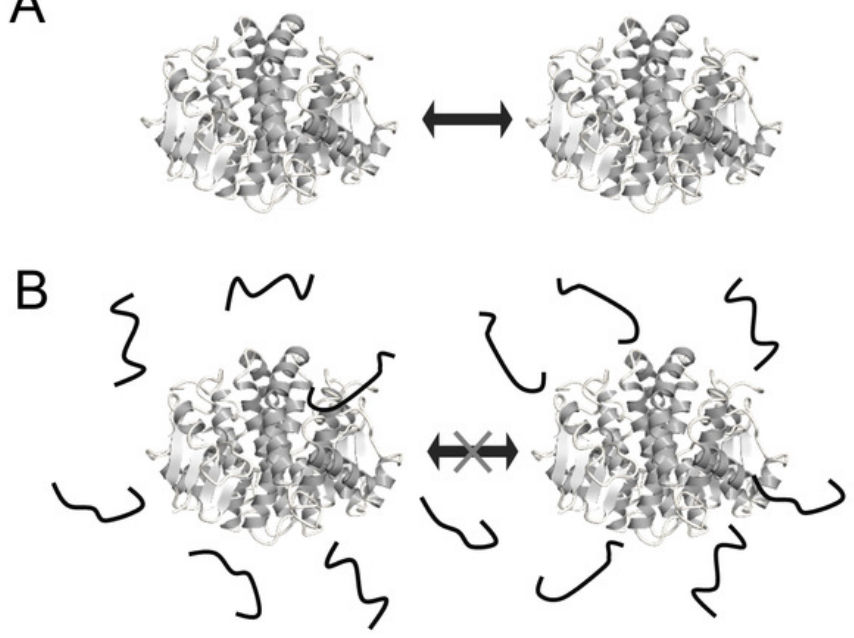